Research Article

\title{
Construction of Consistent Comparison Matrix by Macharis' Method Revisit
}

\author{
Yi-Fong Lin \\ Department of Fashion Industry Management, Hsing Wu University, New Taipei City 25103, Taiwan \\ Correspondence should be addressed to Yi-Fong Lin; 109287@mail.hwu.edu.tw
}

Received 6 January 2021; Revised 17 February 2021; Accepted 12 March 2021; Published 30 March 2021

Academic Editor: Muhammet Gul

Copyright (@ 2021 Yi-Fong Lin. This is an open access article distributed under the Creative Commons Attribution License, which permits unrestricted use, distribution, and reproduction in any medium, provided the original work is properly cited.

\begin{abstract}
A famous paper that has been cited more than four hundred times tried to combine (a) the preference ranking organization method for enrichment evaluations (PROMETHEE) and (b) the analytic hierarchy process (AHP) to construct a new method for multicriteria decision-making problems. The paper developed a consistent comparison matrix for their AHP by the defined first row and then they allowed the expert to change several entries in the comparison matrix. Hence, how to construct a new comparison matrix that is (i) consistent and (ii) satisfying the assigned values by the expert becomes a challenging problem. A recent article provided a reply to the above problem by the construction of all entries for the comparison matrix. However, they did not follow the original design proposed by the famous paper. In this paper, we present a new approach with a proposition that satisfies the original design of the famous paper and also achieves two goals (i) and (ii). The research gap of proof is fulfilled by this paper. Our findings explain that the original construction of the famous paper to develop a consistent comparison matrix only by the first row with several assigned values by an expert is indeed workable under two additional restrictions proposed by the recent article. We believe that after our proposition, researchers have the confidence to execute the original design of the paper that has been cited more than four hundred times.
\end{abstract}

\section{Introduction}

Macharis et al. [1] explained the benefit and deficiency of the PROMETHEE (Brans and Vincke [2], Brans and Mareschal [3], and Brans [4]) and the AHP methods (Saaty [5-8]) and integrate them into an AHP-PROMETHEE method for decision ranking problems with achievements on operational consistency and consensus. Up to now, more than four hundred and seventy papers have cited Macharis et al. [1] in their references. It indicates that the AHP-PROMETHEE method is a hot research problem for decisionmaking problems. Since Chu et al. [9] had already provided a comprehensive literature review for those papers published before 2018, we will only examine those 152 papers published after 2018.

We can classify those 152 papers into the following six categories. In the first categories, those papers discussed the analytic hierarchy process or fuzzy analytical hierarchy process. However, they did not apply the construction proposed by Macharis et al. [1] such that they will not face the problem of changing entries of a consistent matrix by experts. We will not list all of them in the following, but only mention three of them: Çalişkan et al. [10], Farooq et al. [11], and Jamshidi et al. [12].

In the second category, this paper paid attention to the problem of changing entries in a consistent comparison matrix by experts and then adjusting entries to preserve the consistent property. We list him in the following: Chu et al. [9], specifically.

Chu et al. [9] pointed out that Macharis et al. [1] provided a consistent comparison matrix and then informed readers the expert wanted to change three entries. Macharis et al. [1] provided the results for the first two changes, but Macharis et al. [1] did not show how to execute their change. Chu et al. [9] developed their method to satisfy the preassigned value by the expert and still result in a consistent matrix. Moreover, Chu et al. [9] showed that two extra conditions must be kept to run the changing entries of a 
consistent comparison matrix. The detailed explanation of Chu et al. [9] will be presented in Section 2.

In the third category, those papers focused on the realworld application for mining, agriculture, fishery, waste, water, land, and power. Three of them are listed below: Schmitz et al. [13], Zheng and Lienert [14], and Zhao et al. [15]. In the fourth category, those papers dealt with transit issues. We mentioned two of them as follows: Gökçekuş et al. [16], and Barabinoet al. [17].

In the fifth category, those papers considered business problems. We list one of them in the following: Andreopoulou et al. [18]. In the sixth category, those papers developed new methods for multicriteria decision problems. Three papers of them are listed in the following: Marttunen et al. [19], Chelmis et al. [20], and Zhou et al. [21]. Based on the above literature review, we can claim that only Chu et al. [9] pay the attention to the consistent comparison matrix proposed by Macharis et al. [1].

Macharis et al. [1] decided on the first row of a matrix and then used the first row to define the remaining rows to construct a consistent comparison matrix. Macharis et al. [1] then wanted experts to review the consistent comparison matrix to revise several entries. Macharis et al. [1] did not explain how to execute those revisions but provided two examples. Recently, Chu et al. [9] published a paper in Mathematical Problems in Engineering to discuss the revision process for Macharis' consistent algorithm. They pointed out that Macharis et al. [1] did not show the revision process in detail; instead, they only demonstrated the revision process by two examples. Chu et al. [9] used these two examples to develop an algorithm for the revision process and found two conditions to guarantee the process was workable.

Until now, no papers have applied the consistent comparison matrix to handle a practical problem. Hence, there is a need to provide an efficient and understandable algorithm for researchers to apply the proposed consistent comparison matrix of Macharis et al. [1].

Chu et al. [9] is an interesting paper but we find several shortcomings in their paper:

(a) Their definition of the revised new comparison matrix did not follow the original design of Macharis et al. [1] which was developed only by the first row.

(b) Their proof for their proposed matrix being reciprocal, consistent, and attaining the desired value is tedious.

(c) After adding two criteria, Chu et al. [9] only offered an example to illustrate the revision process is workable. We provide a proposition to prove the revision procedure is valid under two criteria proposed by Chu et al. [9].

Therefore, we provide a new construction for the revision process of the comparison matrix of Macharis et al. [1] and Chu et al. [9].

This paper is organized as follows: in Section 2, we provide a brief review for Macharis et al. [1] and Chu et al. [9]. Our developed process is discussed in Section 3.
Comparison between our method and Chu et al. [9] is presented in Section 4. In Section 5, we provide a proposition to show that the revision process is valid under two criteria proposed by $\mathrm{Chu}$ et al. [9] and we also prepare an algorithm for the changing process. In Section 6, a numerical example is shown by an abstract approach to demonstrate its validity. We conclude our paper in Section 7.

\section{Prerequisite from the Review}

We recall the construction of Macharis et al. [1] for their first consistent comparison matrix. First, Macharis et al. [1] decided $a_{1 j}$ for $j=1,2, \ldots, n$, with $a_{11}=1$, and then they assumed

$$
a_{i j}=\frac{a_{1 j}}{a_{1 i}},
$$

for $i, j=1,2, \ldots, n$. After Macharis et al. [1] developed a method to construct a consistent comparison matrix, they invited an expert to select several entries to modify their values. We refer to the original consistent matrix, denoted as $\left[x_{i j}\right]_{8 \times 8}$, proposed by Macharis et al. [1] in Table 1 .

Macharis et al. [1] claimed the expert wanted to change the value of $a_{57}$ from $7 / 5$ to 2 . We quote the second comparison matrix, denoted as $\left[y_{i j}\right]_{8 \times 8}$, proposed by Macharis et al. [1] in Table 2. We follow Chu et al. [9] to mark those unchanged blocks by boldface.

Macharis et al. [1] further mentioned that the expert desired another improvement for $a_{45}$ from $5 / 2$ to 2 . We cite the third comparison matrix proposed by Macharis et al. [1] in Table 3. We follow Chu et al. [9] to mark those unchanged blocks by boldface.

Chu et al. [9] pointed out Macharis et al. [1] did not inform readers how to develop the new comparison matrix after a specific entry is changed. The main purpose of Chu et al. [9] is to offer a reasonable method to show how to construct an improved comparison matrix with a predesigned entry.

Chu et al. [9] assumed the original consistent comparison matrix, which is denoted as $\left[a_{i j}\right]_{n \times n}$ and a specific entry, denoted as $a_{\alpha \beta}$, with $\alpha<\beta$, which will be assigned to a new value $\delta$, and then Chu et al. [9] denoted the revised matrix as $\left[b_{i j}\right]_{n \times n}$ such that the new matrix satisfies three properties: Property (P1):

$$
b_{i j}=a_{i j}, \quad \text { for } 1 \leq i, j \leq \alpha .
$$

Property (P2):

$$
b_{i j}=a_{i j}, \quad \text { for } \alpha+1 \leq i, j \leq n,
$$

and Property (P3):

$$
b_{\alpha \beta}=\delta \text {. }
$$

Properties (P1) and (P2) are related to those blocks marked by boldface in Table 2 and marked by boldface in Table 3.

Chu et al. [9] constructed their new matrix, $\left[b_{i j}\right]_{n \times n}$ item by item for all entries, by the following four rules: 


$$
\begin{gathered}
\text { If } 1 \leq i \leq \alpha, \\
1 \leq j \leq \alpha, \text { then } b_{i j}=a_{i j}, \\
\text { If } 1 \leq i \leq \alpha, \\
\alpha+1 \leq j \leq n, \text { then } b_{i j}=a_{i \alpha} \delta a_{\beta j}, \\
\text { If } \alpha+1 \leq i \leq n, \\
1 \leq j \leq \alpha, \text { then } b_{i j}=a_{i \beta}\left(\frac{1}{\delta}\right) a_{\alpha j}, \\
\text { If } \alpha+1 \leq i \leq n, \\
\alpha+1 \leq j \leq n, \text { then } b_{i j}=a_{i j} .
\end{gathered}
$$

In Theorem 1 of Chu et al. [9], by four different cases, they proved their new matrix is reciprocal; that is, it is a comparison matrix. In Theorem 2 of Chu et al. [9], by eight different cases, they verified their new matrix is consistent. In Theorem 3 of Chu et al. [9], they showed the desired entry is attained.

The construction proposed by Chu et al. [9] is listed in equations (5-8) directly defined for all entries. However, the original design of Macharis et al. [1] is only defined for the first row and then equation (1) is applied for the remaining rows. This observation motivates us to develop a new revision process in the next section.

\section{Methodology and Lemmas}

From a consistent comparison matrix, denoted as $\left[a_{i j}\right]_{n \times n}$ and proposed by Macharis et al. [1], experts wanting to change several entries of $\left[a_{i j}\right]_{n \times n}$, and the resulting comparison matrix being still consistent, Chu et al. [9] tried to find a new consistent comparison matrix, denoted as $\left[b_{i j}\right]_{n \times n}$, with several desired properties. Their approach is interesting and achieves their goal. Their construction is defined for every entry of $\left[b_{i j}\right]_{n \times n}$ with several properties of equations ((2)-(4)). However, Chu et al. [9] did not motivate their construction. Moreover, their construction violated the intent of Macharis et al. [1] to develop a new consistent comparison only by the data of the first row. In this paper, we derive a new method to adjust entries of a consistent comparison matrix and also fulfill the changes by experts. We need four lemmas to prove our new matrix is reciprocal, consistent, and attains the predesign for a specific entry as well as the invariant phenomenon mentioned in Chu et al. [9] as equations (2) and (3). On the contrary, Chu et al. [9] only need three theorems to verify their new matrix as reciprocal, consistent, and attaining the predesigned for a specific entry.

Chu et al. [9] did not need to show their new matrix satisfied the invariant phenomenon mentioned in Chu et al. [9] of equations (2) and (3), because the invariant phenomenon was already built in their item through the item definition of the matrix.

Owing to the construction of Chu et al. [9], during their proof, for each index, denoted as $i$, they must divide into two cases as $i \in\{1,2, \ldots, \alpha\}$ and $i \in\{\alpha+1, \ldots, n\}$ such that when checking the reciprocal condition that involves two indexes $\{i, j\}$, they must then divide their proof into four cases. When checking the consistent condition, that involves three indexes $\{i, j, k\}$ and then they must divide their proof into eight cases.

We will develop a new method with the following features:

(a) Satisfying the original intent of Macharis et al. [1] to use data from the first row to find the entries of other rows.

(b) The proof of our proposed matrix becoming simple.

(c) We show our developed new matrix satisfying the invariant phenomenon mentioned in $\mathrm{Chu}$ et al. [9] of equations (2) and (3), which can be verified with simple proofs.

Therefore, we will follow the intent of Macharis et al. [1] to define our revised comparison matrix such that only the first row is defined by us and then the rest of the entries are defined by equation (1).

We face the following problem:

The original consistent comparison matrix is $\left[a_{i j}\right]_{n \times n}$, to construct a new consistent comparison matrix, denoted as $\left[c_{i j}\right]_{n \times n}$. The $(\alpha, \beta)$ entry will be changed from $a_{\alpha, \beta}$ to $\delta$, with $\alpha<\beta$, that is, $c_{\alpha \beta}=\delta$ such that $c_{i j}=a_{i j}$, for $1 \leq i, j \leq \alpha$ and $c_{i j}=a_{i j}$, for $\alpha+1 \leq i, j \leq n$.

We define the first row as follows:

$$
c_{1 j}=a_{1 j}
$$

for $1 \leq j \leq \alpha$, and

$$
c_{1 j}=a_{1 \alpha} \delta a_{\beta j},
$$

for $\alpha+1 \leq j \leq n$, and then for the rest entries, for $i=2,3, \ldots, n$,

$$
c_{i j}=\frac{c_{1 j}}{c_{1 i}} \text {. }
$$

We know equation (11) also works for $i=1$, since $c_{11}=a_{11}=1 q$. We begin to prove our constructed matrix has the desired properties.

Lemma 1. Our new matrix is reciprocal, that is, $c_{i j} c_{j i}=1$.

Proof. We compute

$$
c_{i j} c_{j i}=\left(\frac{c_{1 j}}{c_{1 i}}\right)\left(\frac{c_{1 i}}{c_{1 j}}\right)=1,
$$


to imply our proposed matrix being a comparison matrix.

Lemma 2. Our new matrix is consistent; that is, $c_{i j} c_{j k}=c_{i k}$.

Proof. We derive

$$
c_{i j} c_{j k}=\left(\frac{c_{1 j}}{c_{1 i}}\right)\left(\frac{c_{1 k}}{c_{1 j}}\right)=\left(\frac{c_{1 k}}{c_{1 i}}\right)=c_{i k} .
$$

Lemma 3. Our new matrix attains the predesigned value, $c_{\alpha \beta}=\delta$.

Proof. We evaluate

$$
c_{\alpha \beta}=\frac{c_{1 \beta}}{c_{1 \alpha}}=\frac{a_{1 \alpha} \delta a_{\beta \beta}}{a_{1 \alpha}}=\delta,
$$

since $\alpha<\beta$ and $a_{\beta \beta}=1$.

In the following, we prove our construction satisfies Properties 1 and 2 .

Lemma 4. $c_{i j}=a_{i j}$, for $1 \leq i, j \leq \alpha$, and $c_{i j}=a_{i j}$, for $\alpha+1 \leq i, j \leq n$.

Proof. We obtain

$$
c_{i j}=\frac{a_{1 j}}{a_{1 i}}=a_{i j},
$$

for $1 \leq i, j \leq \alpha$, because the original matrix $\left[a_{i j}\right]_{n \times n}$ is consistent and

$$
c_{i j}=\frac{a_{1 \alpha} \delta a_{\beta j}}{a_{1 \alpha} \delta a_{\beta i}}=a_{i j},
$$

for $\alpha+1 \leq i, j \leq n$, because the original matrix $\left[a_{i j}\right]_{n \times n}$ is consistent.

\section{Methodology Comparison}

Here, we compare our proof with that of Chu et al. [9]. Our Lemma 1 is related to Theorem 1 of Chu et al. [9]; however, Chu et al. [9] needed four cases for their Theorem 1. Our Lemma 2 is related to Theorem 2 of Chu et al. [9], but Chu et al. [9] needed eight cases for their Theorem 2. Our Lemma 3 is related to Theorem 3 of Chu et al. [9] such that all three proofs are very simple. Our Lemma 4 does not appear in Chu et al. [9], because Chu et al. [9] defined their new matrix $\left[b_{i j}\right]_{n \times n}$ item by item such that Properties P1 and P2, equations (2) and (3), that is our Lemma 4, are already contained in the definition of Chu et al. [9].

However, their item by item construction makes their proof very tedious in many cases. On the other hand, our proofs are elegant and satisfy the original intent of Macharis et al. [1] to define a new matrix only by the new structure of the first row.

In Chu et al. [9], they constructed numerical examples to demonstrate that without their second criterion, further revision will change previously preassigned values. However,
Chu et al. [9] did not provide proof to show that, under their two extra criteria, the revision procedure is executable. In the next section, we will present theoretical proof to verify their two extra criteria are valid for the revision process.

\section{Insights on the Two Criteria in Revision Process}

Table 7 of Chu et al. [9] showed the expert wanted to change values of $a_{57}, a_{26}, a_{15}$, and $a_{24}$, one at a time, and then the satisfaction rate increased $25 \%$ per revision operation for the first three revisions. However, after the fourth revision, the satisfaction rate drops to $50 \%$, indicating the revision procedure of Macharis et al. [1] needs improvements.

We recall Tables (8)-(11) of Chu et al. [9] to indicate if the expert wants to change the values of $a_{24}$ and $a_{26}$, then an infinite repeated revision process will occur and a deadlock phenomenon will appear.

Chu et al. [9] claimed two criteria must be preserved to avoid an endless revision process and we cite these in the following.

The first criterion: the order of changes should be arranged by the descending order of row index. On the contrary, in Macharis et al. [1], the order of changes is arranged by the descending order of the column index.

The second criterion: when the expert decides to select several entries to modify their values one by one, there must only be a maximum of one entry per row that can be modified. On the other hand, Macharis et al. [1] mentioned the expert wanted to change the values of $\left\{a_{26}, a_{24}\right\}$ which are two items in the same row. Hence, Chu et al. [9] claimed the revision procedure of $\left\{a_{57}, a_{26}, a_{15}, a_{24}\right\}$ by the descending order of column index should be improved to $\left\{a_{57}, a_{26}, a_{15}\right\}$ or $\left\{a_{57}, a_{24}, a_{15}\right\}$ by the descending order of the row index.

Chu et al. [9] only used numerical examples to point out a deadlock phenomenon (an endless revision process) that occurs when using the Macharis' approach, but they did not provide proof for their proposed procedure, which is controlled by two criteria mentioned in Chu et al. [9]. In the following, we prove the revision procedure proposed by $\mathrm{Chu}$ et al. (2008) is valid.

We assume in the $m$ th revision step, the expert wanted to change the item for the $(i, j)$ entry to $a_{i j}^{m}=a$ and then in the $(m+1)$ th revision step, the expert wanted to change the item for the $(s, t)$ entry to $a_{s t}^{m+1}=b$ with $i<j, s<t$ and $i>s$, where the comparison matrices are denoted as $\left(a_{i j}^{m}\right)_{n \times n}$ and $\left(a_{i j}^{m+1}\right)_{n \times n}$ for the $m$ th and the $(m+1)$ th revisions. In the previous revision process, $a_{i j}^{m}$ already changed to $a$, and then we will verify that utilizing our proposed method of equations (9)-(11), the new comparison matrix has the desired properties.

Proposition 1. The revision procedure of Chu et al. [9] with two added criteria is valid.

Proof. We develop the new matrix as follows:

$$
a_{1 k}^{m+1}=a_{1 k}^{m},
$$


TABLE 1: Reproduction of Table 5 of Macharis et al. [1], or Table 1 of Chu et al. [9].

\begin{tabular}{ccccccccc}
\hline$D$ & $C_{1}$ & $C_{2}$ & $C_{3}$ & $C_{4}$ & $C_{5}$ & $C_{6}$ & $C_{7}$ \\
\hline$C_{1}$ & 1 & 2 & 3 & 2 & 5 & $2 / 3$ & 7 \\
$C_{2}$ & $1 / 2$ & 1 & $3 / 2$ & 1 & $5 / 2$ & $1 / 3$ & $7 / 2$ \\
$C_{3}$ & $1 / 3$ & $2 / 3$ & 1 & $2 / 3$ & $5 / 3$ & $2 / 9$ & $7 / 3$ \\
$C_{4}$ & $1 / 2$ & 1 & $3 / 2$ & 1 & $5 / 2$ & $1 / 3$ & $7 / 2$ \\
$C_{5}$ & $1 / 5$ & $2 / 5$ & $3 / 5$ & $2 / 5$ & 1 & $2 / 15$ & $7 / 2$ & $7 / 5$ \\
$C_{6}$ & $3 / 2$ & 3 & $9 / 2$ & 3 & $15 / 2$ & 1 & $3 / 5$ \\
$C_{7}$ & $1 / 7$ & $2 / 7$ & $3 / 7$ & $2 / 7$ & $5 / 7$ & $2 / 2$ & $9 / 2$ \\
$C_{8}$ & $1 / 3$ & $2 / 3$ & 1 & $2 / 3$ & $5 / 3$ & $2 / 9$ & 1 \\
\hline
\end{tabular}

TABLe 2: Reproduction of Table 6 of Macharis et al. [1] or Table 2 of Chu et al. [9].

\begin{tabular}{|c|c|c|c|c|c|c|c|c|}
\hline$D$ & $C_{1}$ & $C_{2}$ & $C_{3}$ & $C_{4}$ & $C_{5}$ & $C_{6}$ & $C_{7}$ & $C_{8}$ \\
\hline$C_{1}$ & 1 & 2 & 3 & 2 & 5 & $20 / 21$ & 10 & $30 / 7$ \\
\hline$C_{2}$ & $1 / 2$ & 1 & $3 / 2$ & 1 & $5 / 2$ & $10 / 21$ & 5 & $15 / 7$ \\
\hline$C_{3}$ & $1 / 3$ & $2 / 3$ & 1 & $2 / 3$ & $5 / 3$ & $20 / 63$ & $10 / 3$ & $10 / 7$ \\
\hline$C_{4}$ & $1 / 2$ & 1 & $3 / 2$ & 1 & $5 / 2$ & $10 / 21$ & 5 & $15 / 7$ \\
\hline$C_{5}$ & $1 / 5$ & $2 / 5$ & $3 / 5$ & $2 / 5$ & 1 & $4 / 21$ & 2 & $6 / 7$ \\
\hline$C_{6}$ & $21 / 20$ & $21 / 10$ & $63 / 20$ & $21 / 10$ & $21 / 4$ & 1 & $21 / 2$ & $9 / 2$ \\
\hline$C_{7}$ & $1 / 10$ & $1 / 5$ & $3 / 10$ & $1 / 5$ & $1 / 2$ & $2 / 21$ & 1 & $3 / 7$ \\
\hline$C_{8}$ & $7 / 30$ & $7 / 15$ & $7 / 10$ & $7 / 15$ & $7 / 6$ & $2 / 9$ & $7 / 3$ & 1 \\
\hline
\end{tabular}

for $1 \leq k \leq s$, and

$$
a_{1 k}^{m+1}=a_{1 s}^{m} b a_{t k}^{m}
$$

for $s+1 \leq k \leq n$, and then for the rest entries, for $r=2,3, \ldots, n$, and $k=1,2, \ldots, n$,

$$
a_{r k}^{m+1}=\frac{a_{1 k}^{m+1}}{a_{1 r}^{m+1}} .
$$

From the second half of Lemma 4, we know $a_{r k}^{m+1}=a_{r k}^{m}$, for $s+1 \leq r, k \leq n$. Those previous revisions belong to the block of $(r, k)$ entry, with $s+1 \leq r, k \leq n$, since the revision process is one item at a time and arranged by the descending order of the row index (Criterion 1) and there is at most one entry in each row that can be revised (Criterion 2). Therefore, during the $(m+1)$ th revision, all previous revisions are preserved.

By Lemma 1, our new matrix is a comparison matrix. By Lemma 2, our new comparison matrix is consistent. By Lemma 3, our new consistent comparison matrix satisfies $a_{s t}^{m+1}=b$. Hence, we finish the proof of Proposition 1 .

The following presents an algorithm to help practitioners construct their computing program.

\section{Numerical Example}

After proving the revision procedure satisfying two criteria proposed by $\mathrm{Chu}$ et al. [9] and attaining all wanted properties, we consider the numerical example in Macharis et al. [1] and Chu et al. [9]. Macharis et al. [1] only informed us that $a_{57}$ should be revised from $7 / 5$ to 2 , but they did not tell the values for $a_{15}$ and $a_{24}$. Chu et al. [9] claimed the desired values for $a_{15}$ and $a_{24}$ are 6 and 2, respectively. However, Chu et al. [9] did not show us the evidence as to why they assume the desired values of $a_{15}=6$ and $a_{24}=2$. Consequently, we believe the best way to handle $a_{15}$ and $a_{24}$ is to abstractly assume $a_{24}=A$ and $a_{15}=B$.

We accept the finding of Macharis et al. [1]; that is, the original matrix is shown in Table 1 and $a_{57}$ is revised to 2 in Table 2. Therefore, we list our results for changing to $a_{24}=A$ in Table 4 and for changing to $a_{15}=B$ in Table 5 , respectively. To emphasize those unchanged entries, we marked them by boldface.

Following our algorithm in Section 5, we know that $\mathrm{x}_{\mathrm{ij}}$ is an entry of Table 2, $s=2<t=4$, with $y_{24}=A$, and then $y_{11}=1, y_{12}=2, y_{13}=x_{12} A x_{43}=3 A, y_{14}=x_{12} A x_{44}=2 A$, $y_{15}=x_{12} A x_{45}=5 A, \quad y_{16}=x_{12} A x_{46}=20 A / 21$, $y_{17}=x_{12} A x_{47}=10 A$, and $y_{18}=x_{12} A x_{48}=30 A / 7$. The rest of the entries are computed by $y_{i j}=\left(y_{1 j} / y_{1 i}\right)$, for $i=2,3, \ldots, n$.

From Tables 4 and 5, we illustrate according to the ordering, $a_{57}, a_{24}$, and $a_{15}$ as the descending of the row index and then the desired results of $a_{57}=2, a_{24}=A$, and $a_{15}=B$ are achieved.

When we change the value of $a_{24}$ from 1 to $\mathrm{A}$, then $a_{57}$ is in the low-right bold area in Table 4 such that its value $a_{57}=$ 2 is preserved.

When we change the value of $a_{15}$ from $5 \mathrm{~A}$ to $\mathrm{B}$, then $a_{57}$ and $a_{24}$ are in the low-right bold area such that its values $a_{57}=2$ and $a_{24}=A$ are preserved. 
TABle 3: Reproduction of Table 7 of Macharis et al. [1] or Table 3 of Chu et al. [9].

\begin{tabular}{|c|c|c|c|c|c|c|c|c|}
\hline$D$ & $C_{1}$ & $C_{2}$ & $C_{3}$ & $C_{4}$ & $C_{5}$ & $C_{6}$ & $C_{7}$ & $C_{8}$ \\
\hline$C_{1}$ & 1 & 2 & 3 & 2 & 4 & $21 / 16$ & 8 & $24 / 7$ \\
\hline$C_{2}$ & $1 / 2$ & 1 & $3 / 2$ & 1 & 2 & $8 / 21$ & 4 & $12 / 7$ \\
\hline$C_{3}$ & $1 / 3$ & $2 / 3$ & 1 & $2 / 3$ & $4 / 3$ & $16 / 63$ & $8 / 3$ & $8 / 7$ \\
\hline$C_{4}$ & $1 / 2$ & 1 & $3 / 2$ & 1 & 2 & $8 / 21$ & 4 & $12 / 7$ \\
\hline$C_{5}$ & $1 / 4$ & $1 / 2$ & $3 / 4$ & $1 / 2$ & 1 & $4 / 21$ & 2 & $6 / 7$ \\
\hline$C_{6}$ & $21 / 16$ & $21 / 8$ & $63 / 16$ & $21 / 8$ & $21 / 4$ & 1 & $21 / 2$ & $9 / 2$ \\
\hline$C_{7}$ & $1 / 8$ & $1 / 4$ & $3 / 8$ & $1 / 4$ & $1 / 2$ & $2 / 21$ & 1 & $3 / 7$ \\
\hline$C_{8}$ & $7 / 24$ & $7 / 12$ & $7 / 8$ & $7 / 12$ & $7 / 6$ & $2 / 9$ & $7 / 3$ & 1 \\
\hline
\end{tabular}

(i) Step 1: the previous consistent comparison matrix is denoted as $\left(x_{i j}\right)_{n \times n}$.

(ii) Step 2: the new consistent comparison matrix is denoted as $\left(y_{i j}\right)_{n \times n}$.

(iii) Step 3: the expert wants to change the $(s, t)$ entry to $\Omega$, with $s<t$, that is, $y_{s t}=\Omega$.

(iv) Step 4: assume $y_{1 j}=x_{1 j}$ for $1 \leq j \leq s$.

(v) Step 5: assume $y_{1 j}=x_{1 s} \Omega x_{t j}$ for $s+1 \leq j \leq n$.

(vi) Step 6: for $i=2,3, \ldots, n$, and $1 \leq j \leq n, y_{i j}=y_{1 j} / y_{1 i}$.

(vii) Step 7: the order of changing entries is arranged by the descending order of the row index and there is at most one entry that can be revised.

Algorithm 1

TABle 4: To change $a_{24}$ to $A$.

\begin{tabular}{|c|c|c|c|c|c|c|c|c|}
\hline D & $C_{1}$ & $C_{2}$ & $C_{3}$ & $\mathrm{C}_{4}$ & $C_{5}$ & $C_{6}$ & $C_{7}$ & $C_{8}$ \\
\hline$C_{1}$ & 1 & 2 & $3 \mathrm{~A}$ & $2 \mathrm{~A}$ & $5 A$ & $20 A / 21$ & $10 A$ & $30 A / 7$ \\
\hline$C_{2}$ & $1 / 2$ & 1 & $3 A / 2$ & $A$ & $5 A / 2$ & $10 A / 21$ & $5 A$ & $15 A / 7$ \\
\hline$C_{3}$ & $1 / 3 A$ & $2 / 3 A$ & 1 & $2 / 3$ & $5 / 3$ & $20 / 63$ & $10 / 3$ & $10 / 7$ \\
\hline$C_{4}$ & $1 / 2 A$ & $1 / A$ & $3 / 2$ & 1 & $5 / 2$ & $10 / 21$ & 5 & $15 / 7$ \\
\hline$C_{5}$ & $1 / 5 \mathrm{~A}$ & $2 / 5 A$ & $3 / 5$ & $2 / 5$ & 1 & $4 / 21$ & 2 & $6 / 7$ \\
\hline$C_{6}$ & $21 / 20 A$ & $21 / 10 A$ & $63 / 20$ & $21 / 10$ & $21 / 4$ & 1 & $21 / 2$ & $9 / 2$ \\
\hline$C_{7}$ & $1 / 10 A$ & $1 / 5 A$ & $3 / 10$ & $1 / 5$ & $1 / 2$ & $2 / 21$ & 1 & $3 / 7$ \\
\hline$C_{8}$ & $7 / 30 A$ & $7 / 15 A$ & $7 / 10$ & $7 / 15$ & $7 / 6$ & $2 / 9$ & $7 / 3$ & 1 \\
\hline
\end{tabular}

TABle 5: To change $a_{15}$ to $B$.

\begin{tabular}{|c|c|c|c|c|c|c|c|c|}
\hline$D$ & $C_{1}$ & $C_{2}$ & $C_{3}$ & $C_{4}$ & $C_{5}$ & $C_{6}$ & $C_{7}$ & $C_{8}$ \\
\hline$C_{1}$ & 1 & $2 B / 5 A$ & $3 B / 5$ & $2 B / 5$ & $B$ & $4 B / 21$ & $2 B$ & $6 B / 7$ \\
\hline$C_{2}$ & $5 A / 2 B$ & 1 & $3 A / 2$ & $A$ & $5 A / 2$ & $10 A / 21$ & $5 A$ & $15 A / 7$ \\
\hline$C_{3}$ & $5 / 3 B$ & $2 / 3 A$ & 1 & $2 / 3$ & $5 / 3$ & $20 / 63$ & $10 / 3$ & $10 / 7$ \\
\hline$C_{4}$ & $5 / 2 B$ & $1 / A$ & $3 / 2$ & 1 & $5 / 2$ & $10 / 21$ & 5 & $15 / 7$ \\
\hline$C_{5}$ & $1 / B$ & $2 / 5 A$ & $3 / 5$ & $2 / 5$ & 1 & $4 / 21$ & 2 & $6 / 7$ \\
\hline$C_{6}$ & $21 / 4 B$ & $21 / 10 A$ & $63 / 20$ & $21 / 10$ & $21 / 4$ & 1 & $21 / 2$ & $9 / 2$ \\
\hline$C_{7}$ & $1 / 2 B$ & $1 / 5 A$ & $3 / 10$ & $1 / 5$ & $1 / 2$ & $2 / 21$ & 1 & $3 / 7$ \\
\hline$C_{8}$ & $7 / 6 B$ & $7 / 15 A$ & $7 / 10$ & $7 / 15$ & $7 / 6$ & $2 / 9$ & $7 / 3$ & 1 \\
\hline
\end{tabular}

The above preserving property of previous revisions is proved in our Proposition 1.

\section{Conclusion}

We develop a new method for the Macharis' revision process that fulfills the intent of their design to construct a consistent comparison matrix only using the data from the first row, which is an improvement on a recently published paper. Our method avoids the tedious Chu's construction to define all entries to result in a simpler proof for reciprocal and consistent properties as Lemmas 1 and 2 in our paper. The shortcoming of our method is that we must prove the invariant properties of equations (2) and (3) that is our Lemma 4. However, please refer to our Lemma 4 to understand that it is a straightforward proof to demonstrate the efficiency of our method.

By our Proposition 1, we proved that the revision process obeying two Chu's criteria is valid. Researchers will have the confidence to apply this AHP-PROMETHEE method to their decision-making problems. 


\section{Data Availability}

The inventory model discussed in this paper is cited from Omar et al.'s study [4] that was published in Computing and Industrial Engineering in 2010.

\section{Conflicts of Interest}

The authors declare there are no conflicts of interest regarding the publication of this paper.

\section{Acknowledgments}

This research was partially supported by the Ministry of Science and Technology, R.O.C. (Grant no. MOST 106-2410H-156-009).

\section{References}

[1] C. Macharis, J. Springael, K. De Brucker, and A. Verbeke, "PROMETHEE and AHP: the design of operational synergies in multicriteria analysis," European Journal of Operational Research, vol. 153, no. 2, pp. 307-317, 2004.

[2] J. P. Brans and P. Vincke, "Note-A preference ranking organisation method," Management Science, vol. 31, no. 6, pp. 647-656, 1985.

[3] J.-P. Brans and B. Mareschal, "The PROMCALC \& GAIA decision support system for multicriteria decision aid," Decision Support Systems, vol. 12, no. 4-5, pp. 297-310, 1994.

[4] J.-P. Brans, "The space of freedom of the decision maker modelling the human brain," European Journal of Operational Research, vol. 92, no. 3, pp. 593-602, 1996.

[5] T. L. Saaty, Decision Making for Leaders, Lifetime Learning Publications, Belmont, CA, USA, 1982.

[6] T. L. Saaty, "Axiomatic foundation of the analytic hierarchy process," Management Science, vol. 32, no. 7, pp. 841-855, 1986.

[7] T. L. Saaty, The Analytic Hierarchy Process, McGraw-Hill, New York, NY, USA, 1980.

[8] T. L. Saaty, Decision Making for Leaders, The Analytic Hierarchy Process for Decisions in a Complex World, RWS Publications, Pittsburgh, PA, USA, 1995.

[9] C. H. Chu, S. C. Lin, and P. Julian, "Construction and restrictions of consistent matrixes by Macharis' method," Mathematical Problems in Engineering, vol. 2018, Article ID 5901704, 9 pages, 2018.

[10] E. Çalişkan, E. Aksakal, S. Çetinyokuş, and T. Çetinyokuş, "Hybrid use of Likert scale-based AHP and PROMETHEE methods for hazard analysis and consequence modeling (HACM) software selection," International Journal of Information Technology and Decision Making, vol. 18, no. 5, pp. 1689-1715, 2019.

[11] A. Farooq, M. Xie, S. Stoilova, and F. Ahmad, "Multicriteria evaluation of transport plan for high-speed rail: an application to Beijing-Xiongan," Mathematical Problems in Engineering, vol. 2019, Article ID 8319432, 23 pages, 2019.

[12] A. Jamshidi, F. Jamshidi, D. Ait-Kadi, and A. Ramudhin, "A review of priority criteria and decision-making methods applied in selection of sustainable city logistics initiatives and collaboration partners," International Journal of Production Research, vol. 57, no. 15-16, pp. 5175-5193, 2019.

[13] W. I. Schmitz, D. L. P. Feil, L. N. Canha, A. R. Abaide, T. B. Marchesan, and R. Carraro, "Operational vulnerability indicator for prioritization and replacement of power transformers in substation," International Journal of Electrical Power \& Energy Systems, vol. 102, pp. 60-70, 2018.

[14] J. Zheng and J. Lienert, "Stakeholder interviews with two MAVT preference elicitation philosophies in a Swiss water infrastructure decision: aggregation using SWING-weighting and disaggregation using UTAGMS," European Journal of Operational Research, vol. 267, no. 1, pp. 273-287, 2018.

[15] N. Zhao, Z. Xu, and Z. Ren, "Hesitant fuzzy linguistic prioritized superiority and inferiority ranking method and its application in sustainable energy technology evaluation," Information Sciences, vol. 478, pp. 239-257, 2019.

[16] H. Gökçekuş, D. U. Ozsahin, and D. Al-Othman, "Evaluation of the impact of sustainable transportation alternatives on environment using fuzzy PROMETHEE method," International Journal of Innovative Technology and Exploring Engineering, vol. 8, no. 8, pp. 3254-3259, 2019.

[17] B. Barabino, N. A. Cabras, C. Conversano, and A. Olivo, "An integrated approach to select key quality indicators in transit services," Social Indicators Research, vol. 149, no. 3, pp. 1045-1080, 2020.

[18] Z. Andreopoulou, C. Koliouska, E. Galariotis, and C. Zopounidis, "Renewable energy sources: using PROMETHEE II for ranking websites to support market opportunities," Technological Forecasting and Social Change, vol. 131, pp. 31-37, 2018.

[19] M. Marttunen, V. Belton, and J. Lienert, "Are objectives hierarchy related biases observed in practice? A meta-analysis of environmental and energy applications of multi-criteria decision analysis," European Journal of Operational Research, vol. 265, no. 1, pp. 178-194, 2018.

[20] E. Chelmis, D. Niklis, G. Baourakis, and C. Zopounidis, "Multiciteria evaluation of football clubs: the Greek superleague," Operational Research, vol. 19, no. 2, pp. 585-614, 2019.

[21] S. Zhou, X. Ji, and X. Xu, “A hierarchical selection algorithm for multiple attributes decision making with large-scale alternatives," Information Sciences, vol. 521, pp. 195-208, 2020. 